Correction

\title{
Correction to: "Corrosion-Inhibition Effect of Different Phosphate Compounds for Carbon Steel in Chloride- Contaminated Mortars"'[nt. J. Electrochem. Sci., 14(2019) 8601-8610; doi: 10.20964/2019.09.29]
}

Jing Zhang ${ }^{1}$, Xiangyu $\mathrm{Lu}^{1,2, *}$, Shengli Chen ${ }^{3}$, Leyuan Zhang ${ }^{1}$, Chao Zhu ${ }^{1}$, Yiji Zhang ${ }^{1}$,Tong Wu ${ }^{l}$, Xingguo Feng ${ }^{1,2}$

${ }^{1}$ Jiangsu Key Laboratory of Coast Ocean Resources Development and Environment Security, Hohai University, Nanjing 210098, Jiangsu, China

${ }^{2}$ State Key Laboratory of Geohazard Prevention and Geoenvironment Protection, Chengdu University of Technology, Chendu 610059, Sichuan, China

${ }^{3}$ CNOOC information technology Co., Ltd. Oceanographic information Center, Beijing 100013, China

*E-mail: luxiangyu@hhu.edu.cn

Received: 8 January 2019 / Accepted: 8 January 2019 / Published: 7 October 2019

The authors regret that there were errors in the original published version

The author Shengli Chen and Xingguo Feng, as well as the affiliations of Shengli Chen, "CNOOC information technology Co., Ltd. Oceanographic information Center, Beijing 100013, China", should be added.

The correct paragraph is as follows:

Jing Zhang ${ }^{1}$, Xiangyu Lu $^{1,2, *}$, Shengli Chen ${ }^{3}$, Leyuan Zhang ${ }^{1}$, Chao Zhu ${ }^{1}$, Yiji Zhang ${ }^{1}$,Tong Wu ${ }^{l}$, Xingguo Feng ${ }^{1,2}$

${ }^{1}$ Jiangsu Key Laboratory of Coast Ocean Resources Development and Environment Security, Hohai University, Nanjing 210098, Jiangsu, China

${ }^{2}$ State Key Laboratory of Geohazard Prevention and Geoenvironment Protection, Chengdu University of Technology, Chendu 610059, Sichuan, China

${ }^{3}$ CNOOC information technology Co., Ltd. Oceanographic information Center, Beijing 100013, China *E-mail: luxiangyu@hhu.edu.cn

(C) 2019 The Authors. Published by ESG (www.electrochemsci.org). This article is an open access article distributed under the terms and conditions of the Creative Commons Attribution license (http://creativecommons.org/licenses/by/4.0/). 